\title{
THE EXISTENCE OF CERTAIN ULTRAFILTERS ON $N$ AND A CONJECTURE OF GRAHAM AND ROTHSCHILD
}

\author{
NEIL HINDMAN
}

\begin{abstract}
The equivalence of a conjecture of Graham and Rothschild and the existence of certain ultrafilters on $N$ is established. It is also shown that this conjecture is equivalent to an apparently stronger statement.
\end{abstract}

1. Introduction. Graham and Rothschild have asked [2, p. 291] if, whenever $N$ is divided into two classes, there must always be an infinite subset of one of those classes such that all finite sums of distinct members of that subset remain in the same class. This question is attributed to them as a conjecture by Erdös in [1].

The conjecture came to the author's attention in the form of two questions of Fred Galvin who hoped they might shed some light on this conjecture. They were: (1) Does there exist an ultrafilter $p$ on $N$ such that whenever $A \in p$ one also has $\{x: A+x \in p\} \in p$ ? and (2) Does there exist an ultrafilter $p$ on $N$ such that whenever $A \in p$ one also has $\{x: A-x \in p\} \in$ $p$ ? (Here $A+x=\{y+x: y \in A\}$ and $A-x=\{y-x: y \in A$ and $y>x\}$.) In Galvin's words these would be called almost upward translation invariant and almost downward translation invariant ultrafilters respectively.

The former question is answered in the negative in $\S 4$. In $\S 3$ it is shown, with the aid of the continuum hypothesis, that the second question is in fact equivalent to the Graham-Rothschild conjecture. In $\S 2$ the conjecture is shown to be equivalent to the existence of another ultrafilter on $N$ and to an apparently stronger statement of the conjecture.

2. The Graham-Rothschild conjecture and preliminary results. We write here $A \subseteq{ }_{f} B$ whenever $A$ is a nonempty finite subset of $B$ and $[n, \infty)$ for $\{x: x \in N$ and $n \leqq x\}$.

2.1 Definition. $\Gamma=\{A: A \subseteq N$ and there is an infinite subset $B$ of $A$ such that $\sum F \in A$ whenever $\left.F \subseteq{ }_{f} B\right\}$.

The Graham-Rothschild conjecture can thus be restated as: If $N=A \cup B$ then $A \in \Gamma$ or $B \in \Gamma$. The stronger version referred to above is: If $N=\bigcup_{i=1}^{n} A_{i}$ then there is some $i$ such that $A_{i} \in \Gamma$.

Presented to the Society, January 18, 1972 under the title The nonexistence of a certain ultrafilter on $N$; received by the editors February 17, 1972.

AMS 1969 subject classifications. Primary 1060; Secondary 0460, 5453.

Key words and phrases. Sequences, ultrafilters on $N$, finite distinct sums. 
The following lemma is established by an easy induction.

2.2 LEMMA. If $F$ is a subset of $N$ with $2^{n}$ elements then there is a subset $G$ of $F$ such that $2^{n} \mid \sum G$.

2.3 Lemma. If $A \in \Gamma$ then there exists a sequence $\left\{y_{n}\right\}_{n=1}^{\infty}$ such that $\sum_{n \in F} y_{n} \in A$ whenever $F \subseteq{ }_{f} N$ and such that $2^{r+1} \mid y_{n+1}$ whenever $2^{r} \leqq y_{n}$.

Proof. Let $B$ be as given by the definition of $\Gamma$ and write $B=\left\{x_{n}\right\}_{n=1}^{\infty}$ with $x_{n}<x_{n+1}$ for every $n$. Let $y_{1}=x_{1}$ and assume that for each $m<n$ we have chosen $y_{m}$ and a subset $F_{m}$ of $\left\{x_{n}\right\}_{n=1}^{\infty}$ such that (1) $y_{m}=\sum F_{m}$; (2) if $s<m$ and $x \in F_{s}$ and $y \in F_{m}$ then $x<y$; and (3) if $s<m$ and $2^{r} \leqq y_{s}$ then $2^{r+1} \mid y_{m}$.

Let $x_{k}=\max F_{n-1}$ and let $r$ be the largest integer such that $2^{r} \leqq y_{n-1}$.

Let $a=k+2^{r+1}$ and let $G=\left\{x_{t}\right\}_{t=k+1}^{a}$. Then $G$ has $2^{r+1}$ elements so by Lemma 2.2 there is a subset $F_{n}$ of $G$ such that $2^{r+1} \mid \sum F_{n}$. Let $y_{n}=\sum F_{n}$.

The sequence $\left\{y_{n}\right\}_{n=1}^{\infty}$ is easily seen to satisfy the conclusion of the lemma since $\sum_{n \in F} y_{n}=\sum\left(\bigcup_{n \in F} F_{n}\right)$.

2.4 Lemma. Assume that the Graham-Rothschild conjecture holds and let $\left\{x_{n}\right\}_{n=1}^{\infty}$ be a sequence in $N$ such that $x_{n+1}>\sum_{i=1}^{n} x_{i}$ for every $N$. Let $A=\left\{\sum_{n \in F} x_{n}: F \subseteq_{f} N\right\}$. If $A=B_{1} \cup B_{2}$ then there is an $i$ and $a$ sequence $\left\{y_{n}\right\}_{n=1}^{\infty}$ such that $\left\{\sum_{n \in F} y_{n}: F \subseteq_{f} N\right\} \subseteq B_{i}$ and whenever $F \subseteq_{f}[r, \infty)$ there is $a G \subseteq_{f}[r, \infty)$ such that $\sum_{n \in F} y_{n}=\sum_{n \in G} x_{n}$.

Proof. Define $g: A \rightarrow N$ by the rule $g\left(\sum_{n \in F^{\prime}} x_{n}\right)=\sum_{n \in F} 2^{n-1}$. Since every $x$ in $N$ has a unique binary expansion and since $\sum_{i=1}^{n} x_{i}<x_{n+1}, g$ is one-to-one and onto.

For $i \in\{1,2\}$ let $D_{i}=g\left[B_{i}\right]$. Then $N=D_{1} \cup D_{2}$ so by assumption there is an $i$ such that $D_{i} \in \Gamma$. Let $\left\{z_{n}\right\}_{n=1}^{\infty}$ be the sequence in $D_{i}$ guaranteed by Lemma 2.3. Let $y_{n}=g^{-1}\left(z_{n}\right)$ and note that for each $n$ there exists $G_{n} \subseteq{ }_{f} N$ such that $z_{n}=\sum_{s \in G_{n}} 2^{s}$. Further, since $2^{s+1} \mid z_{n+1}$ whenever $2^{s} \leqq z_{n}$, if $s \in G_{m}$ and $t \in G_{n}$ and $m<n$ then $s<t$. Now $\sum_{s \in G_{n}} 2^{s}=g\left(\sum_{s \in G_{n}} x_{s}\right)$ so $y_{n}=$ $\sum_{s \in G_{n}} x_{s}$. Hence if $F \subseteq_{f} N$ and $G=\bigcup_{n \in F} G_{n}$ then $\sum_{n \in F} y_{n}=\sum_{s \in G} x_{s}$. To complete the proof note that $n \leqq t$ whenever $t \in G_{n}$.

2.5 THEOREM. The Graham-Rothschild conjecture holds if and only if there is an ultrafilter $p$ on $N$ such that $p \subseteq \Gamma$.

Proof. Since any set or its complement must be in $p$ the sufficiency is trivial.

Necessity. Let $p$ be a subset of $\Gamma$ which is maximal with respect to closure under finite intersection. ( $\{N\}$ is a subset of $\Gamma$ closed under finite intersection so we can find such a maximal subset $p$.) 
Suppose $p$ is not an ultrafilter. Then there exist $B_{1}$ and $B_{2}$ such that $N=B_{1} \cup B_{2}$ and $B_{1} \notin p$ and $B_{2} \notin p$. But then there exist $A_{1}$ and $A_{2}$ in $p$ such that $B_{1} \cap A_{1} \notin \Gamma$ and $B_{2} \cap A_{2} \notin \Gamma$. Let $A=A_{1} \cap A_{2}$. Then $A \in p$ so $A \in \Gamma$ and there exists a sequence $\left\{x_{n}\right\}_{n=1}^{\infty}$ such that $x_{n+1}>\sum_{i=1}^{n} x_{i}$ for every $n$ and $\sum_{n \in F}, x_{n} \in A$ whenever $F \subseteq{ }_{f} N$.

Let $D=\left\{\sum_{n \in F} x_{n}: F \subseteq{ }_{f} N\right\}$. Then $D \subseteq A$ so $D \cap B_{1} \subseteq A_{1} \cap B_{1}$ and hence $D \cap B_{1} \notin \Gamma$. Similarly $D \cap B_{2} \notin \Gamma$. But this contradicts the conciusion of Lemma 2.4 .

2.6 COROLlaRY. The Graham-Rothschild conjecture holds if and only if whenever $N=\bigcup_{i=1}^{n} A_{i}$ there is an $i$ such that $A_{i} \in \Gamma$.

3. The conditional existence of an almost downward translation invariant ultrafilter. The ultrafilter produced in Theorem 2.5 does not necessarily answer Galvin's second question even if the Graham-Rothschild conjecture is assumed to be valid. It is in fact possible to produce, under this assumption, an ultrafilter contained in $\Gamma$ which has an element $A$ such that $A-x \notin \Gamma$ for every $x$ in $A$. (To see this let $\left\{B_{n}: n \in N\right\}$ be a partition of $N$ into infinite sets. Let $D_{n}=\left\{\sum_{m \in F} 2^{n}: F \subseteq{ }_{f} B_{n}\right\}$ and let $A_{n}=\bigcup_{k=n}^{\infty} D_{k}$. Each $A_{n} \in \Gamma$ and $\left\{A_{n}: n \in N\right\}$ is closed under finite intersections. There is therefore, as in Theorem 2.5, an ultrafilter $g$ contained in $\Gamma$ and containing $\left\{A_{n}: n \in N\right\}$. But if $x \in A_{1}$ then $x \in D_{n}$ for some $n$ and $\left(A_{1}-x\right) \cap A_{n+1}=\varnothing$.)

3.1 Lemma. Let $p$ be an ultrafilter on $N$. Then $\{x: A-x \in p\} \in p$ whenever $A \in P$ if and only if whenever $A \in p$ there exists $x$ in $A$ such that $A-x \in p$.

Proof. Necessity is trivial.

Sufficiency. Suppose $A \in p$ but $\{x: A-x \in p\} \notin p$. Then, letting $D=\{x: A-x \notin p\}$, one has $D \in p$ and consequently $D \cap A \in p$. Thus there exists $y$ in $D \cap A$ such that $(D \cap A)-y \in p$. But then $A-y \in p$ while $y \in D$, a contradiction.

3.2 Lemma. Let $\left\{x_{n}: n \in N\right\} \subseteq N$ and $\left\{W_{n}: n \in N\right\} \subseteq \mathscr{P}(N)$ such that $x_{n} \in W_{n}$ and $W_{n+1} \subseteq W_{n} \cap\left(W_{n}-x_{n}\right)$ for each $n$. If $F \subseteq_{f}[n, \infty)$ then $\sum_{r \in F} x_{r} \in W_{n}$.

Proof. If $|F|=1$ the result is trivial. Assume the lemma is valid whenever $|F|<m$ and let $|G|=m$ (where $G \subseteq_{f}[n, \infty)$ ). Let $s$ be the first element of $G$ and let $F=G \backslash\{s\}$. Then $|F|<m$ and $F \subseteq_{f}[s+1, \infty)$ so $\sum_{r \in F^{\prime}} x_{r} \in W_{s+1}$ while $W_{s+1} \subseteq W_{s}-x_{s}$. Therefore $x_{s}+\sum_{r \in F^{\prime}} x_{r}=\sum_{r \in G} x_{r} \in W_{s}$ while $s \geqq n$. Thus $\sum_{r \in G} x_{r} \in W_{n}$.

3.3 THEOREM. Assume the continuum hypothesis. The Graham-Rothschild conjecture holds if and only if there exists an ultrafilter $p$ on $N$ such that $\{x: A-x \in p\} \in p$ whenever $A \in p$. 
Proof. Sufficiency. ${ }^{1}$ Let $A \cup B=N$. Without loss of generality $A \in p$. Let $A_{1}=A$ and let $x_{1} \in A_{1}$ such that $A_{1}-x_{1} \in p$. Let $A_{2}=A_{1} \cap\left(A_{1}-x_{1}\right)$. Then $A_{2} \in p$ and we may choose inductively $A_{n}$ and $x_{n}$ in $A_{n}$ such that $A_{n+1} \in p$ where $A_{n+1}=A_{n} \cap\left(A_{n}-x_{n}\right)$. By Lemma 3.2 one has each $A_{n} \in \Gamma$ and in particular $A \in \Gamma$.

Necessity. Well order the power set of $N$ by the ordinals less than $\omega_{1}$, letting $N$ itself appear first in this order. Write $\mathscr{P}(N)=\left\{A_{\alpha}: \alpha<\omega_{1}\right\}$. Let $Z_{0}=A_{0}, \Pi_{0}=\{(n): n \in N\}$ (where $(n)=\{r n: r \in N\}$ ) and assume that for each $\sigma<\alpha$ we have chosen $Z_{\sigma}$ and $\Pi_{\sigma}$ satisfying the following inductive hypotheses.

(1) $Z_{\sigma}=A_{\sigma}$ or $Z_{\sigma}=N \backslash A_{\sigma}$;

(2) $\left|\Pi_{\sigma}\right|=\aleph_{0}$ and if $\tau<\sigma$ then $\Pi_{\tau} \subseteq \Pi_{\sigma}$;

(3) $Z_{\sigma} \in \Pi_{\sigma}$; and

(4) if $\Delta \subseteq{ }_{f} \Pi_{\sigma}$ then there exist an infinite subset $B$ of $\cap \Delta$ and, for each $x$ in $B$, an element $W_{x}$ of $\Pi_{\sigma}$ such that $W_{x} \subseteq \bigcap \Delta \cap(\cap \Delta-x)$.

Inductive hypotheses (1), (2), and (3) clearly hold when $\sigma=0$. To see that (4) holds note that if $\Delta \subseteq_{f} \Pi_{0}$ then $\bigcap \Delta=(n)$ for some $n$ and that $(n) \cap((n)-r n)=(n)$ for every $r$ and $n$.

Now let $\Pi_{\alpha}^{\prime}=\bigcup_{\sigma<\alpha} \Pi_{\sigma}$. Since $\alpha<\omega_{1}$ we have $\left|\Pi_{\alpha}^{\prime}\right|=\aleph_{0}$ so we may write $\Pi_{\alpha}^{\prime}=\left\{V_{n}: n \in N\right\}$. Let $U_{n}=\bigcap_{k=1}^{n} V_{k}$. Now $U_{1} \in \Pi_{\sigma}$ for some $\sigma<\alpha$ so there exist $x_{1}$ and $t$ such that $x_{1} \in U_{1}$ and $V_{t} \subseteq U_{1} \cap\left(U_{1}-x_{1}\right)$. Let $m(1)=1$ and $m(2)=t+1$. In particular then $U_{m(2)} \subseteq U_{m(1)} \cap\left(U_{m(1)}-\dot{x}_{1}\right)$. Assume we have chosen $x_{s}$ and $m(s+1)$ for each $s<r$ such that $x_{s} \in U_{m(s)}, x_{s}>\sum_{k=1}^{s-1} x_{k}$, $m(s+1)>m(s)$, and $U_{m(s+1)} \subseteq U_{m(s)} \cap\left(U_{m(s)}-x_{s}\right)$. Since $\left\{V_{k}: 1 \leqq k \leqq m(r)\right\} \subseteq$ $\Pi_{\tau}$ for some $\tau<\alpha$ and since $U_{m(r)}=\bigcap_{k=1}^{m(r)} V_{k}$ there are an infinite subset $B$ of $U_{m(r)}$ and, for each $x$ in $B$, an element $D_{x}$ of $\Pi_{r}$ such that $D_{x} \subseteq U_{m(r)} \cap$ $\left(U_{m(r)}-x\right)$. Let $x_{r} \in B$ such that $x_{r}>\sum_{k=1}^{r-1} x_{k}$. Then $D_{x_{r}}=V_{n}$ for some $n$. Let $m(r+1)=\max \{n, m(r)+1\}$. Then $U_{m(r+1)} \subseteq U_{m(r)} \cap\left(U_{m(r)}-x_{r}\right)$ as desired.

Let $W_{r}=U_{m(r)}$ and note that $W_{r} \subseteq U_{r}$. By Lemma 3.2 if $F \subseteq_{f}[r, \infty)$ then $\sum_{s \in F} x_{s} \in W_{r}$. By Lemma 2.4 if $B_{1}=A_{\alpha} \cap\left\{\sum_{s \in F} x_{s}: F \subseteq{ }_{f} N\right\}$ and $B_{2}=$ $\left\{\sum_{s \in k^{\prime}} x_{s}: F \subseteq{ }_{f} N\right\} \backslash A_{\alpha}$ then there is an $i$ and a sequence $\left\{y_{n}\right\}_{n=1}^{\infty}$ such that $\left\{\sum_{s \in F} y_{s}: F \subseteq_{f} N\right\} \subseteq B_{i}$ and whenever $F \subseteq_{f}[n, \infty)$ there is a $G \subseteq_{f}[n, \infty)$ such that $\sum_{s \in F} y_{s}=\sum_{s \in G} x_{s}$. In particular if $F \subseteq \subseteq_{f}[r, \infty)$ then $\sum_{s \in F} y_{s} \in W_{r}$ and in fact $\sum_{s \in F} y_{s} \in U_{r}$.

Let $Z_{\alpha}=A_{\alpha}$ if $i=1$ and let $Z_{\alpha}=N \backslash A_{\alpha}$ if $i=2$. Let $S_{n}=\left\{\sum_{s \in F} y_{s}: F \subseteq \subseteq_{f}\right.$ $[n, \infty)\}$ and let $\Pi_{\alpha}=\Pi_{\alpha}^{\prime} \cup\left\{Z_{\alpha}\right\} \cup\left\{S_{n}: n \in N\right\}$. Then hypotheses (1), (2), and (3) are trivially satisfied.

In order to verify hypothesis (4) let $\Delta \subseteq{ }_{f} \Pi_{\alpha}$. Then $\Delta=\Delta_{1} \cup \Delta_{2}$ where $\Delta_{1} \subseteq \Pi_{\alpha}^{\prime}$ and $\Delta_{2} \subseteq\left\{Z_{\alpha}\right\} \cup\left\{S_{n}: n \in N\right\}$ (and not both $\Delta_{1}$ and $\Delta_{2}$ are empty).

\footnotetext{
1 Added in proof. The sufficiency of Theorem 3.3 was observed by Galvin.
} 
If $\Delta_{2}=\varnothing$ then the result is true by assumption since $\Delta_{1} \subseteq \Pi_{\sigma}$ for some $\sigma<\alpha$. If $\Delta_{2} \neq \varnothing$ then there is some $n$ such that $S_{n} \subseteq \bigcap \Delta_{2}$ (for $S_{1} \subseteq Z_{\alpha}$ and $S_{n+1} \subseteq S_{n}$ ). If $\Delta_{1}=\varnothing$ then $S_{n} \subseteq \bigcap \Delta$. If $\Delta_{1} \neq \varnothing$ then $U_{s} \subseteq \bigcap \Delta_{2}$ for some $s$. Since $S_{s} \subseteq U_{s}$ we have in either case some $r$ such that $S_{r} \subseteq \bigcap \Delta$. Let $B=\left\{y_{k}: k \in[r, \infty)\right\}$ and let $y_{k} \in B$. Then $S_{k+1} \in \Pi_{\alpha}$ and $S_{k+1} \subseteq S_{r} \cap$ $\left(S_{r}-y_{k}\right) \subseteq \bigcap \Delta \cap\left(\cap \Delta-y_{k}\right)$ as desired. The induction is complete. Let $p=\bigcup_{\alpha<\omega_{1}} \Pi_{\alpha} \cdot p$ is clearly an ultrafilter.

Now let $A \in p$. To complete the proof it suffices, by Lemma 3.1, to produce $x$ in $A$ such that $A-x \in p . A \in \Pi_{\alpha}$ for some $\alpha$ so by hypothesis (4) there is some $x$ in $A$ and some $W$ in $\Pi_{\alpha}$ such that $W \subseteq A \cap(A-x)$. Then $W \in p$ and $W \subseteq A-x$ so $A-x \in p$ as desired.

What is not answered by Theorem 3.3 is the validity of the GrahamRothschild conjecture. It does relate that conjecture to an ultrafilter, which may be viewed as a point in $\beta N$, the Stone-Cech compactification of $N$. Unfortunately the author has been unable to topologically characterize those points corresponding to almost downward translation invariant ultrafilters.

4. The nonexistence of an almost upward translation invariant ultrafilter on $N$. Galvin's first question is answered in the negative by exhibiting two sets whose union is $N$, neither one of which could be in any ultrafilter with the property that $\{x: A+x \in p\} \in p$ whenever $A \in p$.

4.1 LEMMA. If $p$ is an ultrafilter on $N$ with the property that $\{x: A+x \in p\} \in p$ whenever $A \in p$ then for each element $A$ of $p$ there is a sequence $\left\{x_{n}\right\}_{n=1}^{\infty}$ in $A$ such that for each $n, x_{n+2}>x_{n}+x_{n+1}, x_{n+1}>x_{n}$, and $\left\{x_{n}, x_{n+1}-x_{n}, x_{n+2}-\left(x_{n}+x_{n+1}\right)\right\} \subseteq A$.

Proof. Let $A_{1}=A$ and choose $\left\{x_{n}\right\}_{n=1}^{\infty}$ and $\left\{A_{n}\right\}_{n=1}^{\infty}$ such that $x_{n} \in A_{n}$, $A_{n}+x_{n} \in p$, and $A_{n+1}=A_{n} \cap\left(A_{n}+x_{n}\right)$. Then clearly $\left\{x_{n}, x_{n+1}-x_{n}\right\} \subseteq A$ for each $n$. Also $x_{n+2} \in A_{n+1} \cap\left(A_{n+1}+x_{n+1}\right)$ so $x_{n+2}-x_{n+1} \in A_{n+1}=$ $A_{n} \cap\left(A_{n}+x_{n}\right)$. Thus $x_{n+2}-\left(x_{n}+x_{n+1}\right) \in A_{n} \subseteq A$.

4.2 THEOREM. If $p$ is an ultrafilter on $N$ then there is some element $A$ of $p$ such that $\{x: A+x \in p\} \notin p$.

Proof. Suppose $p$ has the property that $\{x: A+x \in p\} \in p$ whenever $A \in p$. For $i \in\{1,2\}$ let $B_{i}=\left\{3^{n}(3 k+i):\{n, k\} \subseteq N \cup\{0\}\right\}$. Then $N=B_{i} \cup B_{2}$ so there is an $i$ in $\{1,2\}$ such that $B_{i} \in p$.

For each $x$ in $N$ let $f(x)$ be the largest integer $n$ such that $3^{n} \mid x$. Let $\left\{x_{n}\right\}_{n=1}^{\infty}$ be as given by Lemma 4.1. Since $x_{n+1}-x_{n} \in B_{i}$ for each $n$ we have $f\left(x_{n+1}\right) \leqq f\left(x_{n}\right)$. Hence there is an $m$ such that $f\left(x_{m}\right)=f\left(x_{m+1}\right)=f\left(x_{m+2}\right)$. But then $x_{m+2}-\left(x_{m}+x_{m+1}\right) \notin B_{i}$, a contradiction. 


\section{REFERENCES}

1. P. Erdös, Problems and results on combinatorial number theory (preprint).

2. R. L. Graham and B. L. Rothschild, Ramsey's theorem for n-parameter sets, Trans. Amer. Math. Soc. 159 (1971), 257-292.

Department of Mathematics, California State College, los angeles, CALIFORNIA 90034 